\title{
Brain Death
}

National Cancer Institute

\section{Source}

National Cancer Institute. Brain Death. NCI Thesaurus. Code C116340.

Irreversible absence of cortical and brain stem functioning. 